\title{
Primer aislamiento de Candida auris en Chile
}

\author{
María Victoria Moreno', María Elvira Simian², Javier Villarroel³, Luz María Fuenzalida', \\ María Fernanda Yarad ${ }^{1}$, Andrés Soto ${ }^{3}$, Verónica Silva ${ }^{4}$ y Ximena Pimente/ ${ }^{4}$
}

\section{First isolation de Candida auris in Chile}

Candida auris is an emerging multi-drug-resistant fungus that is rapidly spreading worldwide. Since the first reports in 2009, many isolates across five continents have been identified as agents of hospital-associated infections. Independent and simultaneous outbreaks of $C$. auris are becoming a major concern for healthcare and scientific community. Moreover, laboratory misidentification and multi-drug-resistant profiles, rarely observed for other non-albicans Candida species, result in difficult eradication and frequent therapeutic failures of $C$. auris infections. In this article we present the first case of isolation of a strain of C. auris at a hospital in Santiago, in a patient coming from India, who was admitted for treatment of diabetic foot complications. The strain was recovered from a tissue culture and identified by VITEK ${ }^{\circledR} 2$ Compact. The accurate identification of $C$. auris was confirmed by means of MALDI-TOF MS and DNA sequence analysis. The isolate was resistant to fluconazole, retaining only susceptibility to amphotericin and caspofungin with MIC breakpoints recommended by CDC. The emergence of $C$. auris is alarming because the mode of transmission within the healthcare environment is not clear and is likely to be multifactorial.

Keywords: Candida auris; candidemia; invasive fungal infection; antimicrobial resistance; antifungal resistance.

Palabras clave: Candida auris; candidemia; infección fúngica invasora; resistencia antimicrobiana; resistencia antifúngica.

\section{Introducción}

$C$ andida auris es un hongo levaduriforme multiresistente emergente que puede causar infecciones invasoras y que se ha asociado a brotes asociados a la atención de salud alrededor del mundo. Candida auris fue descrita por primera vez en una secreción de oído externo de un paciente japonés en $2009^{1,2}$.

En Chile, no se había reportado hasta la fecha el aislamiento de esta levadura, de acuerdo a reportes de vigilancia de hospitales e Instituto de Salud Pública (ISP). Este es el primer caso comunicado de un aislado de $C$. auris en Chile.

\section{Caso clínico}

Varón de 65 años de edad, de nacionalidad india radicado en Chile. Con diabetes mellitus tipo II hacía seis años, usuario de metformina y atorvastatina. Sin complicaciones conocidas de su patología de base. En agosto de 2018 evolucionó con signos de isquemia y posteriormente con necrosis del cuarto ortejo izquierdo, asociado a celulitis del mismo pie. Sus familiares decidieron su traslado a Bombay, India, para recibir tratamiento. Fue derivado a Inlaks General Hospital de Bombay, donde recibió 14 días de tratamiento antibacteriano con clindamicina y se realizó la amputación del ortejo. En una muestra de secreción purulenta obtenida en la cirugía, se identificó una regular cantidad de cocáceas grampositivas en pares y racimos, sin aislamiento de ningún microorganismo a las $48 \mathrm{~h}$ de incubación. Estuvo internado 24 días por dificultad en el control glucémico. No hay antecedentes de la evolución de la herida operatoria, aunque recibió curaciones en el mismo centro en India por dos meses. Una semana antes de volver a Chile, notó signos compatibles con una necrosis en el tercer ortejo del mismo pie, por lo que al regresar al país consultó en el servicio de urgencia de un centro privado. Fue derivado al Hospital del Salvador, donde se estudió en forma ambulatoria y se derivó a cirugía vascular con diagnóstico de pie diabético con complicaciones vasculares. No se describieron signos de infección.

El 26 de diciembre de 2018 ingresó a pabellón quirúrgico, describiéndose una úlcera plantar en relación a la base del quinto ortejo asociado a una osteítis de la falange distal y proximal del tercer ortejo. Se amputaron ambos ortejos y se tomaron cultivos de tejido del lecho quirúrgico. El paciente fue dado de alta el mismo día con ciprofloxacina y metronidazol por indicación del equipo quirúrgico.

En las muestras de tejido del lecho de amputación del tercer y quinto ortejo no hubo crecimiento de microorga-
'Area Técnica Microbiología, Laboratorio Clínico, Hospital del Salvador, Santiago, Chile. ²Programa de Formación en Microbiología, Facultad de Medicina, Universidad de Chile, Chile.

3Unidad de Infectología, Departamento de Medicina, Hospital del Salvador, Santiago, Chile.

${ }^{4}$ Unidad de Control de Infecciones, Hospital del Salvador, Santiago, Chile.

Conflicto de interés: ninguno Financiamiento: ninguno

Recibido: 20 de junio de 2019 Aceptado: 3 de diciembre de 2019

Correspondencia a: María Victoria Moreno victoria.moreno.lp@gmail.com 
nismos durante las primeras $48 \mathrm{~h}$, por lo que se realizó un traspaso desde el caldo tioglicolato a una placa de agar sangre. El día 29 de diciembre, a las 24 h de incubación, se obtuvo crecimiento de una colonia blanca pequeña que por su aspecto se interpretó como un Staphylococcus spp., realizándose directamente un test de aglutinación que dio un resultado dudoso. El día 31 de diciembre se obtuvo la identificación y susceptibilidad por VITEK ${ }^{\circledR} 2$ Compact: Kokuria kristinae (98\% probabilidad), resistente a aminoglucósidos, quinolonas, eritromicina, clindamicina, teicoplanina y vancomicina. Ante ese perfil de susceptibilidad, se realizó una tinción de Gram en la que se observaron levaduras, por lo que se realizó un traspaso a una placa de agar cromogénico para Candida (BBL CHROMagar Candida Medium) y repetición de la identificación por VITEK $^{\circledR} 2$ Compact. El 1 de enero de 2019 se observó crecimiento de una colonia rosada en el agar cromogénico (Figura 1) y la identificación de levaduras por VITEK ${ }^{\circledR}$ 2 Compact, recientemente actualizado con la versión 8.01, informó Candida auris con $99 \%$ de probabilidad. Dado el resultado obtenido, se realizó identificación por espectrometría de masas MALDI-TOF MS mediante equipo Microflex LT (Bruker Daltonics) a partir de agar Sabouraud (Sabouraud dextrose agar, Biokar diagnostics), confirmándose la identificación de Candida auris (score 1,66). El 3 de enero se envió la cepa al Instituto de Salud Pública (ISP) que confirmó la identificación por secuenciación y reportó el antifungigrama por microdilución en caldo según recomendaciones del (CLSI) (Tabla 1).

Con el resultado del cultivo fue evaluado por Infectología. Sin encontrarse signos inflamatorios en el sitio quirúrgico, no se indicó terapia antimicrobiana ni antifúngica. Tres semanas después de la primera cirugía fue controlado por el equipo de cirugía vascular. Se constató en el sitio quirúrgico salida de exudado purulento con exposición del quinto metatarsiano, sin signos o evidencia de isquemia. El paciente fue sometido a una segunda cirugía el 31 de enero de 2019, realizándose una regularización ósea del

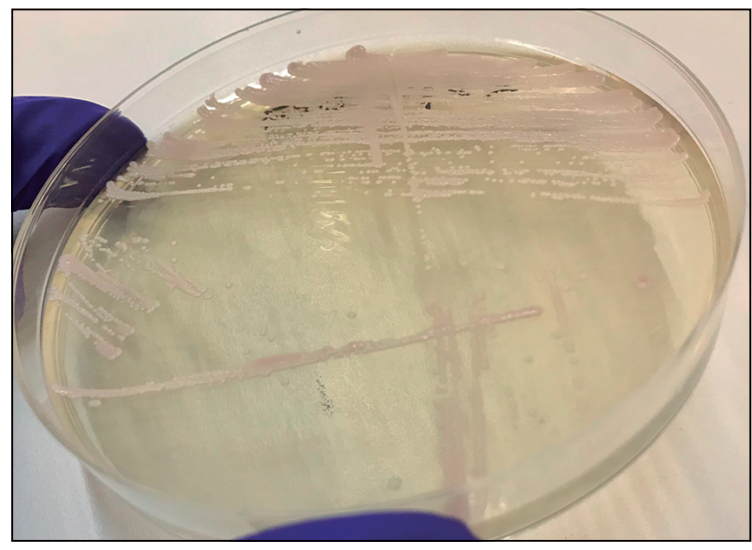

quinto metatarsiano con aseo quirúrgico y obtención de muestras de tejido y hueso. Fue dado de alta el mismo día en buenas condiciones generales, sin sospecha de complicaciones postoperatorias. En estas nuevas muestras, no hubo crecimiento de levaduras, identificándose una Klebsiella pneumoniae en los cultivos de ambas muestras y Enterococcus faecalis y Staphylococcus aureus en la muestra de tejidos blandos. Diez días después fue internado con el diagnóstico de una probable osteomielitis del quinto metatarsiano izquierdo. El 13 de febrero se realizó una amputación transmetatarsiana. Se obtuvieron nuevas muestras para estudio microbiológico de hueso y tejidos blandos del lecho quirúrgico, cuyos cultivos resultaron negativos. El paciente recibió 12 días de vancomicina e imipenem de acuerdo al patrón de susceptibilidad de los agentes identificados, logrando el control del foco infeccioso con la amputación. Dado el tipo de agente identificado, se realizó un estudio de portación nasal, orofaríngeo, axilar, inguino-rectal para la búsqueda de C. auris, que fueron sembrados en placas de agar sangre y tubos de agar Sabouraud. Todos los estudios resultaron negativos. La cepa aislada se envió a los Center for Disease Control and Prevention (CDC) para estudio molecular.

Los datos personales del paciente fueron ingresados a un archivo que se envió al servicio de urgencia y a la unidad de gestión de camas para estar alerta y tomar las medidas correspondientes ante un nuevo ingreso.

Desde que se tuvo conocimiento de la identificación de C. auris, se programó la estadía del paciente en habitación individual con precauciones de contacto, según protocolo institucional. Se informó al personal involucrado, reforzando las medidas de higiene de manos, uso de elementos de protección personal, insumos y equipos individuales, manejo de residuos y el uso en lo posible de material desechable. Se supervisó el cumplimiento de las medidas con pautas de cotejo y de requerir atenciones en otros servicios, como rayos y pabellón, se dio aviso oportuno manteniendo las precauciones de contacto durante todos los procesos.
Figura 1. Candida auris en agar cromogénico Candida.

\begin{tabular}{|c|c|c|}
\hline & $\begin{array}{c}\text { Microdilución } \\
(\mu \mathrm{g} / \mathrm{ml})\end{array}$ & $\begin{array}{c}\text { Interpretación } \\
\text { microdilución }\end{array}$ \\
\hline Anfotericina B & 1 & ND \\
\hline Voriconazol & 0,5 & ND \\
\hline Fluconazol & $>64$ & ND \\
\hline Itraconazol & 0,5 & ND \\
\hline Caspofungina & 0,5 & ND \\
\hline
\end{tabular}


El aseo de la unidad del paciente se realizó dos veces al día con solución de hipoclorito de sodio diluida al 0,5\% (5.000 ppm), como también el aseo terminal de otros servicios como pabellón, que fueron supervisados y controlados con bioluminiscencia. El mismo proceso de aseo y manejo con precauciones de contacto se aplicó para la atención ambulatoria.

\section{Discusión}

\section{Generalidades y dificultades en el diagnóstico}

Candida auris es una especie recientemente reconocida como patógeno nosocomial emergente, causante de brotes en Australia, Canadá, China, Colombia, Francia, Alemania, India, Japón, Kenia, Sudáfrica, Corea del Sur, España, Reino Unido, E.U.A, Panamá y Venezuela, entre otros. En algunos de estos países, la transmisión de C. auris se ha documentado en más de un hospital. Por otro lado, se han notificado casos únicos de C. auris, por ejemplo en países como Austria, Bélgica, Irán, Malasia, Suiza, Taiwán y los Emiratos Árabes Unidos ${ }^{2,3,4}$.

No existen datos de prevalencia de C. auris previo a 2009, de acuerdo a una revisión retrospectiva de un grupo de 15.271 aislados de especies de Candida, incluidos en el programa internacional de vigilancia antifúngica $($ SENTRY) 5 . Sin embargo, desde 2009 ha emergido rápidamente, presentándose como un importante desafío en países como India, donde una serie de Rudramurthy y cols. reportaron 1.400 casos de candidemia, con 74 (5,3\%) casos por $C$. auris, en 19 de 27 UCIs incluidas en el estudio ${ }^{6}$. En Sudamérica, se comunicó el primer brote por C. auris en un hospital terciario en Venezuela, en marzo de 2012, con 18 casos de candidemia por este agente $^{7}$. En general, la prevalencia de $C$. auris sigue en aumento a nivel mundial, con varios brotes reportados ${ }^{4}$.

$\mathrm{Su}$ importancia clínica-epidemiológica radica en ser una especie que presenta multi-resistencia a los antifúngicos y altas tasas de mortalidad asociadas a candidemia, con rangos entre 30 y $60 \%$. Además, se asocia a infecciones prolongadas que ocurren varias semanas después de la admisión, 10 a 50 días, lo que facilita su diseminación en los hospitales ${ }^{8}$.

Otro problema es la dificultad en su identificación, siendo habitualmente informada como Candida haemulonii, Candida sake, Sacharomyces cerevisiae y Rodothorula glutinis por sistemas como VITEK ${ }^{\circledR} 2$ Compact y API20C-AUX. Un 90\% de las cepas son inadecuadamente identificadas por los sistemas bioquímicos comerciales debido a que no tienen esta especie en su base de datos. El diagnóstico de $C$. auris se realiza fundamentalmente mediante secuenciación (regiones ITS o LSU) o por espectrometría de masas MALDI-TOF MS ${ }^{9,10}$.

En relación al problema para su identificación, en nuestro laboratorio el sistema VITEK ${ }^{\circledR} 2$ Compact logró identificar la cepa de $C$. auris a nivel de especie con un $99 \%$ de probabilidad. Esto se explica por la actualización del software con la versión 8.01, la cual incorpora nuevos taxones de identificación, incluyendo C. auris. Los CDC indican que VITEK ${ }^{\circledR} 2$ Compact, con el software versión 8.01, debe ser capaz de detectar con precisión C. auris, aunque se han reportado identificaciones erróneas de ciertos clados, debido a una estrecha relación filogenética con especies del complejo C. haemulonii y por ende toda C. haemulonii (C. haemulonii grupo I) y Candida duobushaemulonii (C. haemulonii grupo II) debe ser enviada a confirmación ${ }^{10}$. Debemos destacar que en nuestro caso hubo un retraso de casi una semana para lograr identificar el agente, debido a que se rescató del traspaso del caldo tioglicolato, una vez que los cultivos originales resultaron negativos con $48 \mathrm{~h}$ de incubación. De este traspaso, se obtuvo una colonia blanca escasa, que fue mal interpretada como una Staphylococcus spp., recordándonos la importancia de la tinción Gram, como un paso clave para dirigir el estudio y que nos habría permitido obtener la identificación por VITEK ${ }^{\circledR} 2$ Compact con una mayor anticipación.

La confirmación por Microflex LT (Bruker) informó el resultado con un bajo score de 1.66, lo que indica que los sistemas MALDI-TOF MS también pueden presentar problemas de identificación, debido a que no todas las bases de datos de referencia incluidos en estos equipos permiten su detección. En un trabajo de Mizusawa y cols. y Ruiz-Gaitán y cols., tanto el sistema MALDI-TOF MS Biotyper $^{\circledR}$ (Bruker, Billerica, MA) como VITEK ${ }^{\circledR}$ MS (bioMérieux) no identificaron los aislados de C. auris o fueron mal identificados como Candida lusitaniae o C. haemulonii, cuando se consultó en las bibliotecas aprobadas por la FDA. La incorporación de una biblioteca de uso exclusivo en investigación (RUO) que contiene $C$. auris, permitió la identificación correcta de este microorganismo por ambos sistemas MALDI-TOF MS. En el sistema VITEK ${ }^{\circledR} \mathrm{MS}$, todos se identificaron correctamente mediante el método de extracción directa con un score cercano al 99\%. Sin embargo, el sistema MALDI Biotyper $^{\circledR}$ de Bruker requiere el método de extracción de tubo completo para lograr un score de identificación cercano o mayor igual a 2,0, porque el método de extracción directa en placa da como resultado coincidencias de puntuación baja para $50 \%$ de los aislamientos de $C$. auris, con un score de $1,7-1,8$ y deja otro $50 \%$ sin identificar ${ }^{11,12}$. Las recomendaciones de los CDC 2018 indican que se debe utilizar el MALDI Biotyper ${ }^{\circledR}$ de Bruker con la biblioteca actualizada Bruker FDA-approved MALDI Biotyper CA System library (Version Claim 4) y el VITEK ${ }^{\circledR}$ MS IVD con la base de datos Saramis Version 3.2 $2^{10,13}$.

Además, los CDC recomiendan el uso de una base de datos complementaria a la de MALDI-TOF, que incluye 
cepas adicionales de $C$. auris de los cuatro clados filogenéticos, que podrían permitir a los usuarios superar los desafíos de identificación, al proporcionar puntuaciones de identificación consistentemente más altas. MicrobeNet es un ejemplo de una base de datos MALDI gratuita en línea, de patógenos inusuales que es verificado por expertos de los CDC. Los usuarios de MicrobeNet pueden tener acceso a bibliotecas de datos espectrales MALDI del CDC para C. auris, así como a la base de datos más actualizada de Bruker (todo gratis con su nombre de usuario creado en MicrobeNet) ${ }^{10}$.

En la actualidad, no se han reportado puntos de corte clínicos para la susceptibilidad de $C$. auris. Los estudios que han examinado la susceptibilidad a este microorganismo han usado una variedad de métodos, incluidos la microdilución en caldo según CLSI, E-test ${ }^{\circledR}$ y VITEK ${ }^{\circledR}$ 2 Compact para levaduras. La concentración inhibitoria mínima (CIM) obtenida para los aislados de C. auris han sido comparados con los puntos de corte determinados para las otras especies de Candida, según tablas del CLSI y EUCAST $^{2}$.

Diversos autores reportan que C. auris tiene baja susceptibilidad a fluconazol (CIM $64 \mu \mathrm{g} / \mathrm{ml}$ ) y susceptibilidad variable a los otros azoles, siendo posaconazol (CIM 0,06$1 \mu \mathrm{g} / \mathrm{ml})$ e isavuconazol (CIM $<0,015-0,5 \mu \mathrm{g} / \mathrm{ml})$ los más activos in vitro ${ }^{8}$. En nuestro caso, no se logró obtener el antifungigrama por VITEK ${ }^{\circledR} 2$ Compact y la susceptibilidad enviada por el ISP fue realizada por microdilución en caldo según las recomendaciones en los documentos M-27,M-60 y M-59 del CLSI. El reporte mostró una CIM elevada a fluconazol (> $64 \mu \mathrm{g} / \mathrm{ml})$, considerado también resistente para las otras especies de Candida. La CIM a voriconazol e itraconazol fue de $0,5 \mu \mathrm{g} / \mathrm{ml}$, que para algunas especies corresponde a categoría intermedia, siendo considerados resistentes CIM $\geq 2 \mu \mathrm{g} / \mathrm{ml}$ para voriconazol $\mathrm{y} \geq 1 \mu \mathrm{g} / \mathrm{ml}$ para itraconazol ${ }^{8,14}$. La CIM de anfotericina B fue de $1 \mu \mathrm{g} / \mathrm{ml}$, sensible para otras especies de Candida y para caspofungina de $0,5 \mu \mathrm{g} / \mathrm{ml}$, para la cual los puntos de corte según la especie de Candida puede ser tanto sensible como resistente ${ }^{14}$. La CIM para micafungina $\mathrm{y}$ anidulafungina no fueron estudiadas. Estos hallazgos concuerdan con los datos de otros investigadores, quienes también han reportado que los aislados de $C$. auris son generalmente resistentes o menos susceptibles a los azoles pero sensibles a equinocandinas.

Los CDC proponen puntos de corte en función de los establecidos para otras especies de Candida estrechamente relacionadas y según la opinión de expertos. El punto de corte para fluconazol considerado resistente es una $\mathrm{CIM} \geq 32 \mu \mathrm{g} / \mathrm{ml}$, demostrándose que los aislados con este valor tienen una mutación en el gen Erg11, siendo poco probable que respondan al tratamiento. Para voriconazol y otros azoles de segunda generación, no proponen puntos de corte e indican considerar la susceptibilidad a fluconazol, pero que la decisión de tratar con estos antifúngicos debe evaluarse caso a caso. Con estudios en modelos de infección por $C$. auris en ratón, definen como punto de corte resistente para anfotericina $\mathrm{B}$ una $\mathrm{CIM} \geq 2 \mu \mathrm{g} / \mathrm{ml}$. En el caso de las equinocandinas, proponen como punto de corte para anidulafungina $\mathrm{y}$ micafungina una $\mathrm{CIM} \geq 4 \mu \mathrm{g} / \mathrm{ml}$, sin puntos de corte del CLSI para estos antifúngicos y para caspofungina una $\mathrm{CIM} \geq 2 \mu \mathrm{g} / \mathrm{ml}$. La correlación entre los puntos de corte microbiológicos y los resultados clínicos no se conoce en este momento. Por este motivo, los puntos de corte propuestos deben considerarse como una guía general y no como definitivos para la resistencia, teniendo en consideración que el hallazgo de una CIM elevada para un antifúngico, no debe necesariamente impedir su uso, especialmente si el uso de otros antifúngicos para el paciente no ha sido efectivo ${ }^{13,15,16}$.

\section{Infección versus colonización}

En el caso presentado, el paciente no recibió tratamiento antifúngico, debido a que en los cultivos de control no hubo crecimiento de $C$. auris, siendo interpretado como una colonización de la piel por este agente. De acuerdo con Schelenzel y cols. que reportan el primer brote por $C$. auris en un hospital en Londres, la mayoría de los casos (56\%) fueron colonización de piel y mucosas, definiendo colonización como cultivo positivo de piel, orofaringe, sitios de salida de dispositivos vasculares, tracto respiratorio y tracto urinario sin signos clínicos de infección por Candida ${ }^{17}$. La colonización por C. auris ha sido detectada en múltiples sitios anatómicos, incluyendo fosas nasales, región axilar, inguinal y rectal y ha sido aislado por más de tres meses después de la detección inicial, a pesar de estudios de portación negativos y tratamiento con equinocandinas. Los factores de riesgo para la colonización incluyen el contacto con pacientes portadores de $C$. auris o su entorno. Se sugiere que el tiempo de contacto para la adquisición de $C$. auris a partir de pacientes colonizados o del ambiente es de menos de $4 \mathrm{~h}$ y en infecciones invasoras es adquirido dentro de las primeras $48 \mathrm{~h}$ desde la admisión a cuidados intensivos. Los aislamientos provenientes de sitios como tracto respiratorio, urinario, piel, tejidos blandos y aparato genital pueden representar una colonización en lugar de infección ${ }^{13,16}$.

Como en otras especies de Candida spp., la presencia de signos y síntomas de infección en el sitio donde se ha aislado $C$. auris puede ayudar a diferenciar entre una colonización e infección. Es importante identificar $C$. auris incluso de un sitio no estéril, porque la colonización plantea riesgo de transmisión, que requiere implementar precauciones de control de infecciones ${ }^{2,16,18}$.

La mayoría de los casos de infección reportados en los últimos cinco años fueron aislados de sangre, pero 
ha sido implicado como agente causal de ventriculitis, osteomielitis, otitis maligna y otomastoiditis, infecciones intra-abdominales complicadas, pericarditis, derrames pleurales y vulvovaginitis. Los factores de riesgo para la infección por C. auris son similares a los asociados a infecciones invasoras por otras Candida spp., que incluyen la exposición previa o continua a antimicrobianos de amplio espectro y antifúngicos, diabetes mellitus, cirugía vascular o abdominal, presencia de dispositivos como catéteres venosos centrales o urinarios, drenajes post-operatorios, enfermedad renal crónica, transfusiones, hemodiálisis, nutrición parenteral total, inmunocompromiso y estadía prolongada en unidad de cuidado intensivo (UCI). La incidencia de C. auris es significativamente mayor en pacientes con alteraciones de la respuesta inmune o secundaria al manejo terapéutico de patologías onco-hematológicas, trasplante de precursores hematopoyéticos y otras condiciones que requieren del uso de agentes inmunosupresores ${ }^{18,19}$.

\section{Rol en infecciones asociadas a la atención de salud}

Candida auris es considerado una amenaza a la salud pública, por su capacidad para originar brotes nosocomiales, lo cual está asociado a dificultades para ser identificado en los laboratorios, su habilidad para persistir en el ambiente hospitalario y superficies de equipos médicos, su capacidad para formar biopelículas $\mathrm{y}$ transmitirse persona a persona por contacto directo y por su resistencia a antifúngicos y a una amplia gama de productos desinfectantes como ácido acético, alcohol y amonio cuaternario, que se utilizan habitualmente en los hospitales ${ }^{20}$.

En estudios ambientales, $C$. auris ha sido aislado de superficies como manguitos de los esfigmomanómetros, mesas, teclados y bombas de infusión en el entorno de la cama del paciente, en unidades de cuidados intensivos quirúrgicos y médicos, como también se ha evidenciado su presencia en los manguitos de presión de pabellón quirúrgico $^{12}$.

Se ha demostrado que $C$. auris sobrevive hasta 14 días en superficies plásticas no porosas secas, similares a las que se encuentran en el entornos de cuidado de salud de los pacientes. En la piel y otros sitios anatómicos puede colonizar durante semanas y meses. Para evitar la transmisión se requieren estrictas medidas de control y prevención:

- Habitación individual con precauciones estándar y de contacto.

- Énfasis en la adherencia a la higiene de manos.

- Limpieza y desinfección ambiental y de equipos reutilizables con los productos recomendados (limpieza diaria y terminal).

- Comunicación entre centros si el paciente es trasladado.
- Estudio de contactos para identificar nuevos casos o colonización por $C$. auris.

- Vigilancia de nuevos casos para detectar transmisión en curso.

Actualmente, los CDC recomiendan continuar con las precauciones de contacto mientras la persona esté colonizada con $C$. auris y realizar evaluaciones periódicas cada tres meses en pacientes colonizados conocidos. La información es limitada sobre la duración de la colonización; sin embargo, la evidencia sugiere que los pacientes permanecen colonizados durante muchos meses, quizás indefinidamente. Se recomienda estudio de portación, al menos con hisopado axilar e inguinal y búsqueda en otros sitios con cultivos positivos anteriores (por ejemplo, orina y esputo). Aunque los pacientes hayan sido colonizados con $C$. auris en nariz, boca, canales auditivos, orina, heridas y recto, estos sitios suelen ser menos sensibles para la detección de colonización. El paciente no debe estar recibiendo antifúngicos activos contra $C$. auris en el momento de estas evaluaciones, de lo contrario se debe esperar una semana y después de la administración de algún antiséptico tópico, por ejemplo clorhexidina, se deben esperar $48 \mathrm{~h}$ antes de realizar cualquier estudio de colonización. Si los hisopados del paciente son positivos, no es necesario repetir el estudio hasta después de tres meses y en el caso que este sea negativo es necesario repetir el estudio en una semana, antes de descontinuar las precauciones de control de infecciones ${ }^{21}$.

La limpieza y desinfección de las habitaciones de los pacientes y el equipo móvil es necesaria para reducir el riesgo de transmisión. Para esto, los CDC recomiendan el uso de desinfectantes registrados en la Agencia de Protección Ambiental (EPA-Environmental Protection Agency, según sus siglas en inglés) que son efectivos contra las esporas de Clostridioides difficile. Un estudio preliminar de la eficacia germicida de ciertos desinfectantes contra C. auris, demuestra que el uso de hipoclorito de sodio al $0,5 \%(5.000 \mathrm{ppm})$ y alcohol $70^{\circ}$, producen una reducción de $4 \log 10$ para C. auris ${ }^{22}$.

Se recomienda el estudio de colonización por C. auris a todo paciente que haya estado internado en un centro de salud en un país con casos documentados de C. auris y también si presentan infección o colonización con bacilos gramnegativos productores de carbapenemasas, debido a que se ha observado la co-colonización regular de ambos. En este momento, no existen pruebas comerciales para el estudio de colonización por C. auris ${ }^{21}$.

Los CDC recomiendan la vigilancia activa prospectiva de laboratorio, identificando las especies de todos los aislamientos de Candida de cualquier fuente de muestra (sitios normalmente estériles y no estériles) de pacientes que estén en la unidad donde se detectó C. auris durante 
al menos un mes, hasta que no haya evidencia de transmisión de C. auris.

La prevención de la transmisión de $C$. auris requiere la estricta adhesión a las recomendaciones de control de infecciones por parte de todo el personal de atención médica que atiende al paciente. Para mejorar la adherencia a las medidas de control de infecciones, se deben considerar los siguientes pasos:

- Educar a todo el personal de atención médica, incluido el personal que trabaja con los servicios de limpieza ambiental y la necesidad de tomar las precauciones adecuadas.

- Asegurarse de que haya suministros adecuados disponibles para implementar las medidas de control de infecciones.

- Supervisar la adherencia a las prácticas de control de infecciones e implementar la limpieza supervisada de las áreas de atención al paciente.

- "Marcar" el registro (ficha) del paciente para instituir las medidas de control de infecciones recomendadas en caso de reingreso ${ }^{21}$.

Agradecimientos: Al personal de apoyo, T.M. Oscar Giaverini, Juan Tapia, Héctor Leiva, Alvaro Arriagada, Víctor Inostroza, Víctor Bahamonde y técnicos paramédicos del área técnica de microbiología del Hospital del Salvador. Agradecer al laboratorio de microbiología del Hospital del Tórax por su apoyo con el equipo Microflex LT (Bruker) y a María Cristina Díaz por sus recomendaciones y contacto con los CDC.

\section{Resumen}

Candida auris es una levadura multi-resistente emergente con rápida diseminación mundial. Desde el primer reporte el 2009, varios aislados a través de los cinco continentes han sido identificados como agentes de infecciones asociadas a la atención en salud. Brotes independientes y simultáneos por $C$. auris se han vuelto prioridad para la comunidad hospitalaria y científica. Además, los errores en identificación y los perfiles de multi-resistencia, raramente observados para otras especies de Candida, resultan en una difícil erradicación y fallas terapéuticas frecuentes en infecciones por C. auris. Presentamos el primer aislamiento de una cepa de C. auris en un hospital en Santiago, en un paciente proveniente de la India, que fue admitido para tratamiento de su pie diabético. La cepa fue recuperada de un cultivo de tejido e identificada por VITEK ${ }^{\circledR} 2$ Compact. La identificación de $C$. auris fue confirmada por MALDI-TOF MS y secuenciación. El aislado fue resistente a fluconazol y susceptible a anfotericina y caspofungina, según puntos de corte recomendados por el CDC. La emergencia de $C$. auris es alarmante debido a que el modo de transmisión dentro del ambiente hospitalario no es claro y probablemente es multifactorial.

\section{Referencias bibliográficas}

1.- Satoh K, Makimura K, Hasumi Y, Nishiyama Y, Uchida K, Yamaguchi H, et al. Candida auris sp. nov., a novel ascomycetous yeast isolated from the external ear canal of an inpatient in a Japanese hospital. Microbiol Immunol 2009; 53: 41-4. doi: 10.1111/j.13480421.2008.00083.x.

2.- Jeffery-Smith A, Taori S K, Schelenz S, Jeffery K, Johnson E M, Borman A, et al. Candida auris: a review of the literature. Clin Microbiol Rev. 2017; 31 (1). pii: e00029-17. doi: 10.1128/ CMR.00029-17.

3.- Araúz A B, Cáceres D H, Santiago E, Armstrong P, Arosamena S, Ramos C, et al. Isolation of Candida auris from 9 patients in Central America: Importance of accurate diagnosis and susceptibility testing. Mycoses 2018; 61: 44-47. doi:10.1111/myc. 12709.

4.- Centers for Disease Control and Prevention. Fungal Diseases. Candida auris. Tracking Candida auris. Disponible: https://www.cdc. gov/fungal/candida-auris/tracking-c-auris.html. Fecha de acceso:

5.- Lockhart S R, Etienne K A, Vallabhaneni S, Farooqi J, Chowdhary A, Govender N P, et al.
Simultaneous emergence of multidrug-resistant Candida auris on 3 continents confirmed by whole-genome sequencing and epidemiological analyses. Clin Infect Dis 2017; 64: 134-40. doi: 10.1093/cid/ciw691.

6.- Rudramurthy S M, Chakrabarti A, Paul R A, Sood P, Kaur H, Capoor M R, et al. Candida auris candidaemia in Indian ICUs: analysis of risk factors. J Antimicrob Chemother 2017; 72: 1794-1801. doi: 10.1093/jac/dkx034.

7.- Calvo B, Melo A S, Perozo-Mena A, Hernández M, Francisco E C, Hagen F, et al. First report of Candida auris in America: Clinical and microbiological aspects of 18 episodes of candidemia. J Infect 2016; 73: 369-74. doi: 10.1016/j.jinf.2016.07.008.

8.- Chowdhary A, Sharma C, Meis J F. Candida auris: A rapidly emerging cause of hospitalacquired multidrug-resistant fungal infections globally. PLoSPathog 2017; 13(5): e1006290. doi: 10.1371/journal.ppat.1006290.

9.- Sears D, Schwartz B S. Candida auris: An emerging multidrug-resistant pathogen. Int J Infect Dis 2017; 63: 95-8. doi:10.1016/j. ijid.2017.08.017.

10.- Centers for Disease Control and Prevention. Fungal Diseases. Candida auris. Identification of Candida auris. Disponible en: https://www. cdc.gov/fungal/candida-auris/recommendations. html. Fecha de acceso:

11.- Mizusawa M, Miller H, Green R, Lee R, Durante M, Perkins R, et al. Can MultidrugResistant Candida auris be reliably identified in clinical microbiology laboratories?. J Clin Microbiol. 2017; 55: 638-40. doi: 10.1128/ JCM.02202-16.

12.- Ruiz-Gaitán A C, Cantón E, Fernández-Rivero M E, Ramírez P, Pemán J. Outbreak of Candida auris in Spain: A comparison of antifungal activity by three methods with published data. Int J Antimicrob Agents. 2019; 53: 541-6. doi: 10.1016/j.ijantimicag.2019.02.005.

13.- Bidaud A L, Chowdhary A, Dannaoui E. Candida auris: An emerging drug resistant yeast - A mini-review. J Mycol Med. 2018; 28: 568-73. doi: $10.1016 / \mathrm{j}$. mycmed.2018.06.007

14.- Clinical and Laboratory Standards Institute. Performance Standards for Antifungal Susceptibility Testing of Yeasts. Approved standard M60. 2017.

15.- Centers for Disease Control and Prevention. Fungal Diseases. Candida auris. Antifungal Susceptibility Testing and Interpretation. 
Disponible en: https://www.cdc.gov/fungal/ candida-auris/c-auris-antifungal.html. Fecha de acceso:

16.- Navalkele B D Revankar S, Chandrasekar P. Candida auris: a worrisome globally emerging pathogen. Expert Rev Anti Infect Ther 2017; 15: 819-27. doi: 10.1080/14787210.2017.1364992.

17.- Schelenz S, Hagen F, Rhodes J L, Abdolrasouli A, Chowdhary A, Hall A, et al. First hospital outbreak of the globally emerging Candida auris in a European hospital. Antimicrob Resist Infect Control 2016; 5: 35. doi:10.1186/s13756016-0132-5.
18.- Cortegiani A, Misseri G, Fasciana T, Giammanco A, Giarratano A, Chowdhary A, et al. Epidemiology, clinical characteristics, resistance, and treatment of infections by Candida auris. J Intensive Care 2018; 6: 69. doi:10.1186/s40560-018-0342-4.

19.- Osei Sekyere J. Candida auris: A systematic review and meta-analysis of current updates on an emerging multidrug-resistant pathogen. Microbiologyopen. 2018; 7: e00578. doi: $10.1002 / \mathrm{mbo} 3.578$.

20.- Sherry L, Ramage G, Kean R, Borman A, Johnson E M, Richardson M D, et al. Biofilm-forming capability of highly virulent, multidrug-resistant Candida auris. Emerg Infect Dis. 2017; 23: 328-31. doi:10.3201/ eid2302.161320.

21.- Centers for Disease Control and Prevention. Fungal Diseases. Candida auris. Infection Prevention and Control for Candida auris. Disponible: https://www.cdc.gov/fungal/ candida-auris/c-auris-infection-control.html. Fecha de acceso:

22.- Rutala W A, Kanamori H, Gergen M, Sickbert-Bennett E, Weber D J. A preliminary study of germicidal efficacy against Candida auris. Open Forum Infect Dis. 2017; 4 (Suppl 1): S184. doi:10.1093/ofid/ofx163.340. 